\title{
Antiassociative groupoids
}

\author{
${ }^{1}$ Milton Braitt \\ ${ }^{2}$ David Hobby \\ ${ }^{2}$ Donald Silberger
}

\begin{abstract}
Given a groupoid $\langle G, \star\rangle$, and $k \geq 3$, we say that $G$ is antiassociative iff for all $x_{1}, x_{2}, x_{3} \in G, \quad\left(x_{1} \star x_{2}\right) \star x_{3}$ and $x_{1} \star\left(x_{2} \star x_{3}\right)$ are never equal. Generalizing this, $\langle G, \star\rangle$ is $k$-antiassociative iff for all $x_{1}, x_{2}, \ldots x_{k} \in G$, any two distinct expressions made by putting parentheses in $x_{1} \star x_{2} \star x_{3} \star \cdots x_{k}$ are never equal.

We prove that for every $k \geq 3$, there exist finite groupoids that are $k$-antiassociative. We then generalize this, investigating when other pairs of groupoid terms can be made never equal.
\end{abstract}

\section{Introduction}

Around fifteen years ago, the second two authors started to investigate finite groupoids which were antiassociative. Instead of obeying the associative law that $\left(x_{1} \star x_{2}\right) \star x_{3}$ and $x_{1} \star\left(x_{2} \star x_{3}\right)$ are always equal, a groupoid is antiassociative iff $\left(x_{1} \star x_{2}\right) \star x_{3}$ and $x_{1} \star\left(x_{2} \star x_{3}\right)$ are never equal. This is a natural change to make to the associative law.

We were aided by a program written by Ming Lei Wu, which went through all the $4^{16}$ possible 4-element groupoids and returned a list of 421,560 which were antiassociative. About $97 \%$ of these antiassociative groupoids were what we called "deranged", and turned out to be constructible in the following way.

Let $G$ be any set with 2 or more elements. First pick a function $f: G \rightarrow G$ with the property that $f(x) \neq x$ for all $x$ (the "derangement"). Then define the binary operation on $G$ by $x \star y=f(x)$, or alternatively, by $x \star y=f(y)$. This makes $\langle G, \star\rangle$ a deranged groupoid. When $x \star y=f(x)$, we have $\left(x_{1} \star x_{2}\right) \star x_{3}=f\left(x_{1}\right) \star x_{3}=f\left(f\left(x_{1}\right)\right) \neq f\left(x_{1}\right)=x_{1} \star\left(x_{2} \star x_{3}\right)$, showing $\langle G, \star\rangle$ is antiassociative. If $x \star y=f(y)$, the proof is similar.

Of the remaining $3 \%$ of the antiassociative groupoids found by the program, almost all had $\star$ tables which were within a few entries of the table of one of the deranged groupoids. But beyond that, we found few patterns in their construction. We conjecture that a similar situation holds for the examples we give in this paper. They probably will not be unique, since it will sometimes be possible to modify them slightly in a haphazard way. 
Before moving on to $k$-antiassociative groupoids, we will invest in some definitions. Using terminology from universal algebra (see [3]), an algebra is a set with some number of (finitary) operations on it. A term of an algebra is any expression on a finite number of variables that can be made by composing the (basic) operations of the algebra. We will use the same notation both for terms as formal expressions and for the resulting functions on an algebra, since the distinction should be clear from context. This paper will focus on groupoids, which are algebras with a single binary operation. We believe that many of our techniques can be used for algebras with multiple operations of any arity, but will not pursue this avenue here.

An ordered term on the variables $x_{j}, x_{j+1}, x_{j+2}, \ldots x_{j+k-1}$ is a $k$-ary term where each variable appears once, in order of their indices. For clarity, we give an inductive definition. Any single variable $x_{j}$ is a 1-ary ordered term. Now suppose that $f$ is an $m$-ary basic operation and that $t_{1}, \ldots t_{m}$ are ordered terms on the variables $x_{j}, x_{j+1}, \ldots x_{j+n-1}$ respectively. (That is, $t_{1}$ is a $k_{1}$-ary ordered term on $x_{j}, x_{j+1}, \ldots x_{j+k_{1}-1}, t_{2}$ is a $k_{2}$-ary ordered term on the next $k_{2}$ variables, and so on, where $n=k_{1}+k_{2}+\cdots k_{m}$.) Then $f\left(t_{1}, t_{2}, \ldots t_{m}\right)$ is an $n$-ary ordered term on the variables $x_{j}, x_{j+1}, \ldots x_{j+n-1}$. We used ordered terms in groupoids in our earlier papers [1] and [2] and called them formal products.

Focusing on groupoids with operation $\star$, we see that there are exactly 5 different ordered terms on the 4 variables $x_{1}, x_{2}, x_{3}, x_{4}$. They are: $\left(\left(x_{1} \star\right.\right.$ $\left.\left.x_{2}\right) \star x_{3}\right) \star x_{4}, \quad\left(x_{1} \star\left(x_{2} \star x_{3}\right)\right) \star x_{4}, \quad\left(x_{1} \star x_{2}\right) \star\left(x_{3} \star x_{4}\right), \quad x_{1} \star\left(\left(x_{2} \star x_{3}\right) \star x_{4}\right)$ and $x_{1} \star\left(x_{2} \star\left(x_{3} \star x_{4}\right)\right)$. As is well known (see [10]), a groupoid has $C(k-1)=(2 k-2) ! / k !(k-1)$ ! many distinct ordered terms on $k$ variables, where $C(m)$ is the $m$-th Catalan number.

Assume $k \geq 3$. Let $s\left(x_{1}, \ldots x_{k}\right)$ and $t\left(x_{1}, \ldots x_{k}\right)$ be distinct terms of some groupoid $\langle G, \star\rangle$. If $s\left(x_{1}, \ldots x_{k}\right) \neq t\left(x_{1}, \ldots x_{k}\right)$ for all $x_{1}, x_{2}, \ldots x_{k} \in$ $G$, then we say that $G$ separates $s$ and $t$. The groupoid $\langle G, \star\rangle$ is $k$ antiassociative iff it separates all the distinct pairs of ordered terms on $x_{1}, x_{2}, \ldots x_{k}$.

Two observations are in order. If $G$ is a groupoid that separates two terms $s$ and $t$, then every subgroupoid of $G$ also separates $s$ and $t$. Second, suppose $G$ is a groupoid that separates $s$ and $t$, and let $H$ be an arbitrary groupoid (with the same operation symbol). Then the Cartesian product $G \times H$ separates $s$ and $t$.

There are infinite groupoids that are $k$-antiassociative for all $k$. One example is $\left\langle F^{\sigma} ; \odot\right\rangle$, the set of all formal products under a natural operation which is similar to concatenation. (See [1] for a definition and proof.) The free groupoid (see [3]) on one or more generators is another example, as can be shown by a modification of the proof of Theorem 3.5. (At the end of the 
proof, where Theorem 3.4 is invoked, one argues directly instead.)

There are no finite groupoids which are $k$-antiassociative for all $k$, since the number of $k$-ary ordered terms increases without bound. Once there are more terms than elements in the groupoid, the Pigeonhole Principle implies that there are terms which will not be separated in the groupoid. This brings us to the following question, which we posed in [2].

Question 1.1. For all $k \geq 3$, is there a finite groupoid that is $k$ antiassociative?

By our observation above, this question may be reduced to the following one.

Question 1.2. For each $k \geq 3$ and for all distinct ordered terms $s$ and $t$ on $x_{1}, x_{2}, \ldots x_{k}$, is there a finite groupoid that separates $s$ and $t$ ?

An affirmative answer to the second question gives an affirmative answer to the first. To see this, assume that for all distinct ordered terms $s$ and $t$ on $x_{1}, x_{2}, \ldots x_{k}$, there is a finite groupoid $G_{s, t}$ that separates $s$ and $t$. Then the product of these groupoids separates all the $k$-ary ordered terms, and is $k$-antiassociative. The other direction is immediate, so the two questions are equivalent.

Note also that whenever $3 \leq j<k$, a groupoid $\langle G, \star\rangle$ that is $k$-antiassociative is also $j$-antiassociative. For suppose $s\left(x_{1}, x_{2}, \ldots x_{j}\right)$ and $t\left(x_{1}, x_{2}, \ldots x_{j}\right)$ are $j$-ary ordered terms that are not separated in $\langle G, \star\rangle$. We let $r\left(x_{j+1}, \ldots x_{k}\right)$ be some fixed $(k-j)$-ary ordered term, and form $s^{\prime}\left(x_{1}, x_{2}, \ldots x_{k}\right)=s\left(x_{1}, x_{2}, \ldots x_{j}\right) \star$ $r\left(x_{j+1}, \ldots x_{k}\right)$ and $t^{\prime}\left(x_{1}, x_{2}, \ldots x_{k}\right)=t\left(x_{1}, x_{2}, \ldots x_{j}\right) \star r\left(x_{j+1}, \ldots x_{k}\right)$. These are two $k$-ary ordered terms that are not separated in $\langle G, \star\rangle$, a contradiction.

$\S 2$ will present two preliminary examples. We will answer Question 1.2 in the affirmative in $\S 3$, and generalize it to arbitrary groupoid terms in $\S 4$.

\section{Preliminary examples}

We start with two simple constructions that often yield groupoids separating two distinct $k$-ary ordered terms. The first is to simply take products of deranged operations. For example, define the operation $\mathrm{L}_{2}$ on the universe of $Z_{2}$ by setting $x \mathrm{~L}_{2} y=(x+1) \bmod 2$. Then we have $\left(x \mathrm{~L}_{2} y\right) \mathrm{L}_{2} z=(x+2)$ $\bmod 2,\left(\left(x \mathrm{~L}_{2} y\right) \mathrm{L}_{2} z\right) \mathrm{L}_{2} w=(x+3) \bmod 2$, and so on. The value of a term with leftmost variable $x$ is $(x+n) \bmod 2$, where $n$ is the depth of $x$ in the term. We also define $\mathrm{R}_{3}$ on the universe of $Z_{3}$ by setting $x \mathrm{R}_{3} y=(y+1)$ mod 3. Similarly, we have that the value of a term with rightmost variable $z$ is $(z+n) \bmod 3$, where $n$ is the depth of $z$ in the term. 
We consider the five possible 4-ary ordered terms, which we list as follows:

$t_{1}=\left(\left(x_{1} \star x_{2}\right) \star x_{3}\right) \star x_{4}$,

$t_{2}=\left(x_{1} \star\left(x_{2} \star x_{3}\right)\right) \star x_{4}$,

$t_{3}=\left(x_{1} \star x_{2}\right) \star\left(x_{3} \star x_{4}\right)$,

$t_{4}=x_{1} \star\left(\left(x_{2} \star x_{3}\right) \star x_{4}\right)$ and

$t_{5}=x_{1} \star\left(x_{2} \star\left(x_{3} \star x_{4}\right)\right)$.

In $\left\langle Z_{2}, \mathrm{~L}_{2}\right\rangle$, we have $t_{1}(w, x, y, z)=\left(w_{1}+3\right) \bmod 2, t_{2}(w, x, y, z)=$ $\left(w_{1}+2\right) \bmod 2, t_{3}(w, x, y, z)=\left(w_{1}+2\right) \bmod 2, t_{4}(w, x, y, z)=\left(w_{1}+1\right)$ $\bmod 2$ and $t_{5}(w, x, y, z)=\left(w_{1}+1\right) \bmod 2$, so all the terms in $\left\{t_{1}, t_{4}, t_{5}\right\}$ are separated from those in $\left\{t_{2}, t_{3}\right\}$ in this groupoid. Similarly, the terms in the sets $\left\{t_{1}, t_{2}\right\},\left\{t_{3}, t_{4}\right\}$ and $\left\{t_{5}\right\}$ are all separated from those in the other sets in the groupoid $\left\langle Z_{3}, \mathrm{R}_{3}\right\rangle$. Continuing, all five terms are separated from each other in the product of the two groupoids.

The problem with this approach is that the value of a term only depends on the depths of its leftmost and rightmost variables, so terms that have those two variables at the same depth can not be separated this way.

The next construction partially avoids this problem. Suppose that $A=$ $\langle A,+\rangle$ is an abelian group, that $\alpha$ and $\beta$ are endomorphisms of $\langle A,+\rangle$, and that $c$ is a fixed element of $A$. We define an operation $\star$ on $A$ by setting $x \star y=\alpha(x)+\beta(y)+c$, and call the groupoid $\langle A, \star\rangle$ the affine endomorphism groupoid for $A, \alpha, \beta$ and $c$. We denote this groupoid by $E(A, \alpha, \beta, c)$.

As an example, suppose we want an affine endomorphism groupoid that separates the terms $s(v, w, x, y, z)=((v \star w) \star(x \star y)) \star z$ and $t(v, w, x, y, z)=$ $((v \star(w \star x)) \star y) \star z$. In both terms, $v$ has depth 3 and $z$ has depth 1 , so the previous approach can't succeed.

In $E(A, \alpha, \beta, c)$, we get $s(v, w, x, y, z)=((\alpha(v)+\beta(w)+c) \star(\alpha(x)+$ $\beta(y)+c)) \star z=\left(\alpha^{2}(v)+\alpha \beta(w)+\alpha(c)+\beta \alpha(x)+\beta^{2}(y)+\beta(c)+c\right) \star z=$ $\alpha^{3}(v)+\alpha^{2} \beta(w)+\alpha^{2}(c)+\alpha \beta \alpha(x)+\alpha \beta^{2}(y)+\alpha \beta(c)+\alpha(c)+\beta(z)+c$. This is quite messy, so we make the simplifying assumptions that $\alpha^{3}=\alpha^{2}$, that $\beta^{2}=\beta$, and that $\alpha$ and $\beta$ commute. This gives us $s(v, w, x, y, z)=\alpha^{2}(v)+$ $\alpha^{2} \beta(w)+\alpha^{2} \beta(x)+\alpha \beta(y)+\beta(z)+\alpha^{2}(c)+\alpha \beta(c)+\alpha(c)+c$. And a similar calculation gives $t(v, w, x, y, z)=\alpha^{2}(v)+\alpha^{2} \beta(w)+\alpha^{2} \beta(x)+\alpha \beta(y)+\beta(z)+$ $\alpha^{2} \beta(c)+\alpha^{2}(c)+\alpha(c)+c$.

Observe that both terms have the identical portion $\alpha^{2}(v)+\alpha^{2} \beta(w)+$ $\alpha^{2} \beta(x)+\alpha \beta(y)+\beta(z)$, and only differ in their constants. (Our choice of simplifying assumptions was designed to do this.) So we can separate the terms by insuring that $\alpha^{2}(c)+\alpha \beta(c)+\alpha(c)+c$ and $\alpha^{2} \beta(c)+\alpha^{2}(c)+\alpha(c)+c$ have different values.

Fortunately, there are $A, \alpha, \beta$ and $c$ that satisfy these conditions. We may work over $\mathbf{Z}_{2}$, and consider $2 \times 3$ matrices with elements in $\mathbf{Z}_{2}$. This 
gives us that the group $A$ is isomorphic to $\mathbf{Z}_{2}^{6}$, a 64-element group. The desired actions of $\alpha$ and $\beta$ on $A$ can be realized by letting $\beta$ copy the top row of $A$ onto the bottom row, and by letting $\alpha$ copy the left column of $A$ onto the middle column and the middle column onto the right column.

That is, $\alpha\left[\begin{array}{lll}d & e & f \\ g & h & i\end{array}\right]=\left[\begin{array}{lll}d & d & e \\ g & g & h\end{array}\right]$ and $\beta\left[\begin{array}{lll}d & e & f \\ g & h & i\end{array}\right]=\left[\begin{array}{lll}d & e & f \\ d & e & f\end{array}\right]$.

Finally, we take $c=\left[\begin{array}{lll}1 & 0 & 0 \\ 0 & 0 & 0\end{array}\right]$. This gives $\alpha \beta(c)=\left[\begin{array}{lll}1 & 1 & 0 \\ 1 & 1 & 0\end{array}\right]$ and $\alpha^{2} \beta(c)=\left[\begin{array}{lll}1 & 1 & 1 \\ 1 & 1 & 1\end{array}\right]$, so $s(\overrightarrow{0})=\left[\begin{array}{lll}0 & 1 & 1 \\ 1 & 1 & 0\end{array}\right]$ and $t(\overrightarrow{0})=\left[\begin{array}{lll}0 & 1 & 0 \\ 1 & 1 & 1\end{array}\right]$.

The above technique requires making assumptions about $\alpha$ and $\beta$ in order to simplify the expressions for the terms. One has some latitude with the assumptions. For example, one may take $\alpha^{k+1}=\alpha^{k}$, or $\beta^{k+1}=\beta^{k}$ for any value of $k$, and no longer require that $\alpha$ and $\beta$ commute. But a point is reached where that no longer helps. We were unable to use the above method to produce a groupoid that separated the two 5-ary terms $s=$ $\left(x_{1} \star\left(x_{2} \star x_{3}\right)\right) \star\left(x_{4} \star x_{5}\right)$ and $t=\left(x_{1} \star x_{2}\right) \star\left(\left(x_{3} \star x_{4}\right) \star x_{5}\right)$. (These terms are represented by trees in Figure 1)

So we turn to another method, which we will present in the next section.

\section{$3 \quad$ Finite $k$-antiassociative groupoids}

We will use a somewhat involved construction, and will require some preliminary definitions. Recall that a full binary tree is a rooted tree where every internal node has exactly two children. (For further definitions and theorems, see [8] or a recent text in discrete mathematics or data structures.)

When full binary trees are used as data structures, the two nodes directly below each internal node are called its left and right children, and the subtrees with these children as roots are the left and right subtrees of that node. As is well known, groupoid terms correspond to full binary trees with leaves labeled by variables. If $s$ is a groupoid term, we will denote the corresponding tree by $T(s)$. This correspondence may be defined recursively as follows. If $s$ is a single variable $x_{i}$, then $T(s)$ is a tree with one node, labelled $x_{i}$. If $s$ and $t$ are groupoid terms, then $T(s \star t)$ is the tree with a root that has $T(s)$ as its left subtree and $T(t)$ as its right subtree.

We will also label the nodes of binary trees with strings made from the characters ' $l$ ' and ' $r$ '. As is usual, we will write the set of all such strings 
as $\{l, r\}^{*}$. In dealing with strings, we will show concatenation by simply writing the two strings next to each other. We use $\Lambda$ to denote the empty string, which is the identity for concatenation. Our labeling may be defined recursively as follows.

The root is labeled $\Lambda$. If a node is labeled $a$, then its left and right children are labeled $a l$ and ar, respectively. These labels may be thought of as directions for how to get to a node by starting at the root and turning the correct way at each branching.

Given a string $p$, an initial substring of $p$ is a string $q$ so that $p=q u$ for some string $u$. (Note that the empty string $\Lambda$ is an initial substring of every string.) A substring is proper if it is not equal to the entire original string, and nontrivial if it is not equal to $\Lambda$.

Putting these two ideas together, occurrences of variables in a groupoid term $s$ correspond to leaves of $T(s)$. The string that is the label of the leaf corresponding to an occurrence of the variable $x_{i}$ will be called the path of that occurrence. If $x_{i}$ only occurs once, we may also call this the path of $x_{i}$. Generalizing this, for any subterm $b$ of $s$, we have that the path of $b$ is also the label of the interior node of $T(s)$ corresponding to the root of subtree $T(b)$.

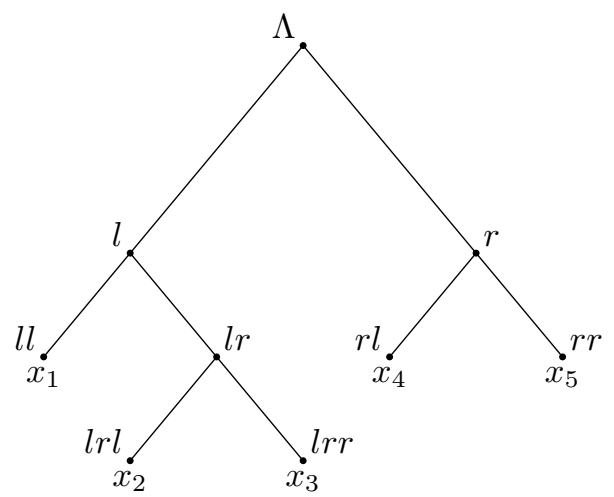

$\left(x_{1} \star\left(x_{2} \star x_{3}\right)\right) \star\left(x_{4} \star x_{5}\right)$

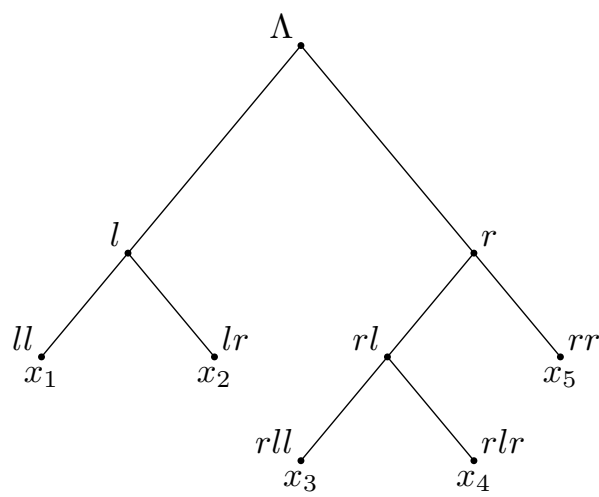

$\left(x_{1} \star x_{2}\right) \star\left(\left(x_{3} \star x_{4}\right) \star x_{5}\right)$

Figure 1: Trees for two terms

For example, consider $s=\left(x_{1} \star\left(x_{2} \star x_{3}\right)\right) \star\left(x_{4} \star x_{5}\right)$. We have $\operatorname{path}\left(x_{1}\right)=$ $l l, \operatorname{path}\left(x_{2}\right)=l r l, \operatorname{path}\left(x_{3}\right)=l r r, \operatorname{path}\left(x_{4}\right)=r l, \quad \operatorname{path}\left(x_{5}\right)=r r$ and $\operatorname{path}\left(x_{2} \star x_{3}\right)=l r$. (When there is danger of confusion, we will write $\operatorname{path}_{s}\left(x_{i}\right)$ to show we mean the path in the term $s$.) The tree for this term is on the left side of Figure 1. 
If $s$ is a groupoid term, we use $\operatorname{Paths}(s)$ for the set of all paths to variables in $s$. Similarly, we have paths to the internal nodes of the tree $T(s)$; these correspond to proper initial substrings of paths to the leaves of $T(s)$. Given the term $s$ with $q$ the path to a node of $T(s)$, we let $s_{q}$ denote the subterm of $s$ with $T\left(s_{q}\right)$ rooted at the node $q$ of $T(s)$. Then for any subterm $b$ of $s$, if we let $q$ be the path of $b$ in $s$, we have $b=s_{q}$.

Our long-term goal is to form a groupoid that separates any two distinct $k$-ary ordered terms $s$ and $t$. We will need some preliminary ideas in order to do this. Our groupoids will have elements which are vectors of finite length over the 2-element field $\mathbf{Z}_{2}$. We take the index set of the components of these vectors to be the set of natural numbers $\mathbf{N}=\{0,1,2, \ldots\}$. All of our vectors will only have a finite number of components, or equivalently, will be vectors indexed by $\mathbf{N}$ that are zero in all but finitely many components. Given any finite set of such vectors, we let $M$ be the set of all indices where any of them have nonzero components. Then all these vectors lie in the finite subspace consisting of vectors with all their components outside of $M$ equal to 0 . We will usually leave this final reduction to a finite groupoid to the reader.

We will actually be using only the additive structure of the field $\mathbf{Z}_{2}$, and viewing it as an abelian group. Our groupoids will all be affine endomorphism groupoids, although the endomorphisms will be built up from their actions on the components of vectors. One nice consequence of this is that we will be able to add groupoid operations pointwise. If $\star_{1}$ and $\star_{2}$ are two groupoid operations on vectors over $\mathbf{Z}_{2}$, their sum $\star_{1}+\star_{2}$ will be defined by $\vec{x}\left(\star_{1}+\right.$ $\left.\star_{2}\right) \vec{y}=\left(\vec{x} \star_{1} \vec{y}\right)+\left(\vec{x} \star_{2} \vec{y}\right)$. Since we are working over $\mathbf{Z}_{2}$, all additions of values such as the above are done modulo 2 . We will periodically note this fact, but not always.

We will define groupoid operations by their actions on components. In this section we will use the convention that the vectors $x, y$ and $z$ are such that $z=x \star y$ for our groupoid operation $\star$. We will also simply write $x$ instead of $\vec{x}$, and write $x[a]$ for the $a$-th component of the vector $x$. (For clarity, we will always use square brackets for this.) To specify a groupoid operation, it then suffices to say what $z[i]$ is for all $i$. We will do this by giving a sequence of equations for the $z[i]$. To emphasize that values are being assigned to the $z[i]$, we will use $:=$ instead of the normal equality symbol. One further convention is that each $z[i]$ will be zero, unless that $z[i]$ is explicitly assigned a value.

For example, consider the groupoid operation which we will later call $\|2, \operatorname{lr}, 0\|$. We define it by the two equations $z[0]:=x[a]$ and $z[a]:=y[2]$. The only indices mentioned are $0, a$ and 2 , so we can focus on just those three components, and view our vectors as 3 -tuples. Writing our operation 
as $\star$, we have $\langle x[0], x[a], x[2]\rangle \star\langle y[0], y[a], y[2]\rangle=\langle x[a], y[2], 0\rangle$. Continuing to use $\star$ for this operation, consider the term $s=(u \star v) \star w$. We have $u \star v=\langle u[a], v[2], 0\rangle$, and $(u \star v) \star w=\langle u[a], v[2], 0\rangle \star\langle w[0], w[a], w[2]\rangle=$ $\langle v[2], w[2], 0\rangle$. The 0 -th component of $s$ is the 2nd component of $v$, where $\operatorname{path}_{s}(v)=l r$. This motivates calling the operation $\|2, l r, 0\|$.

When using the operation $\|2, l r, 0\|$, we will be looking only at the 0 th component of the output, and ignoring the $a$-th component. With this understanding, it makes little difference what the index $a$ is. So we will assume that indices such as $a, b$ and so on are always chosen to minimize collisions. This means that no indices will be equal unless they are explicitly represented with equivalent expressions. This can be easily achieved by appropriate choices of values for $a, b$ and so on, and will not jeopardize the finiteness of any groupoids we produce. As long as there are no collisions, groupoids obtained for different values of $a$ will be isomorphic. Accordingly, we will speak of the groupoid operation $\|2, l r, 0\|$, and so on.

Definition 3.1. Let $p=p_{0} p_{1} p_{2} \cdots p_{j}$ be a nonempty string in $\{l, r\}^{*}$, and let $m$ and $n$ be natural numbers. Then the operation $\|m, p, n\|$ is defined via the following equations, where we assume that $a, a+1, \ldots a+j+1$ are distinct from $m$ and $n$. If $p_{0}$ is $l$, the first equation is $z[n]:=x[a]$, and if $p_{0}$ is $r$, it is $z[n]:=y[a]$. If $p_{1}=l$, the next equation is $z[a]:=x[a+1]$, and if $p_{1}=r$, it is $z[a]:=y[a+1]$. This pattern continues, with $z[a+i]:=x[a+i+1]$ if $p_{i+1}=l$ or $z[a+i]:=y[a+i+1]$ if $p_{i+1}=r$, for all $i \leq j-2$. The last equation is $z[a+j-1]:=x[m]$ if $p_{j}=l$ and it is $z[a+j-1]:=y[m]$ if $p_{j}=r$.

The idea is that $\|m, p, n\|$ transfers the value of the $m$-th component of the vector with path $p$ in the term $s$ to the $n$-the component of the result of $s$, with as few side effects as possible. We are assuming that none of the indices used to define $\|m, p, n\|$ is equal to any of the others, except that possibly $m=n$. In other words, the operation $\|m, p, n\|$ is duplicate free. If $m_{1}$ is distinct from both $m_{2}$ and $m_{0}$, and $p$ and $q$ are strings in $\{l, r\}^{*}$, then the operation $\left\|m_{2}, q, m_{1}\right\|+\left\|m_{1}, p, m_{0}\right\|$ is duplicate free by our convention that indices are chosen to minimize collisions. In isolation, the sum $\left\|m_{2}, q, m_{1}\right\|+\left\|m_{1}, p, m_{0}\right\|$ is equivalent to $\left\|m_{2}, p q, m_{0}\right\|$. The one difference is that the former explicitly mentions the index $m_{1}$. We will henceforth assume that all our groupoid operations are duplicate free.

Lemma 3.2. Let $\star$ be a duplicate and collision free groupoid operation that contains $\|m, p, n\|$ as a summand, and let $s$ be a groupoid term where $p$ is the path to a node of $T(s)$. Letting $s_{p}$ be the subterm of $s$ at that node, $s[n]=s_{p}[m]$ for all values of the variables of $s$. 
Proof. Since $\star$ is duplicate and collision free, the only summand of $\star$ that affects the value of $s[n]$ is $\|m, p, n\|$. So we may ignore the rest of $\star$, and assume $\star$ is $\|m, p, n\|$. Letting $p=p_{0} p_{1} p_{2} \cdots p_{j}$, we will prove the lemma by induction on $j$. Our basis is when $j=0$, making the operation $\left\|m, p_{0}, n\right\|$. We will do the case where $p_{0}=l$, the one for $p_{0}=r$ is similar. Now $s=s_{l} \star s_{r}$, where $\star$ is $\|m, l, n\|$. The one relevant assignment is $z[n]:=x[m]$, giving $s[n]=z[n]=x[m]=s_{l}[m]$, as desired.

For the induction step, assume the statement is true for $j-1$, and that we want to show it for the path $p=p_{0} p_{1} p_{2} \cdots p_{j}$. We write $\star=\|m, p, n\|$ as $\left\|m, p_{j}, b\right\|+\left\|b, p_{0} p_{1} p_{2} \cdots p_{j-1}, n\right\|$ for some new index $b$, and let $q$ be $p_{0} p_{1} \cdots p_{j-1}$, so $p=q p_{j}$. By the statement for $j-1, s[n]=s_{q}[b]$. We have $s_{q}[b]=\left(s_{q l} \star s_{q r}\right)[b]=\left(s_{q l}\left\|m, p_{j}, b\right\| s_{q r}\right)[b]$, where the last step follows because indices are chosen to minimize collisions. There are now two cases. We will do the one for $p_{j}=r$; the case for $p_{j}=l$ is similar. Since $p_{j}=r$, we have $z[b]:=y[m]$ in $\left\|m, p_{j}, b\right\|$. So $s_{q}[b]=s_{q r}[m]=s_{p}[m]$, since $q r=q p_{j}=p$. Thus $s[n]=s_{q}[b]=s_{p}[m]$, as desired.

Given the groupoid operation $\|m, p, n\|$, we define the tweaked operation $\|m, p, n\|^{\prime}$ to be identical to $\|m, p, n\|$ except for one assignment. Writing $p$ as $p_{0} q,\|m, p, n\|$ has an assignment of the form $z[n]:=x[k]$ if $p_{0}=l$ and one of the form $z[n]:=y[k]$ if $p_{0}=r$. Whichever one occurs, we modify it by adding 1 , giving $z[n]:=(x[k]+1) \bmod 2$ if $p_{0}=l$ or giving $z[n]:=(y[k]+1) \bmod 2$ if $p_{0}=r$.

A slight modification of the proof of the previous lemma then establishes the following.

Lemma 3.3. Let $\star$ be a duplicate and collision free groupoid operation that contains $\|m, p, n\|^{\prime}$ as a summand, and let $s$ be a groupoid term where $p$ is the path to a node of $T(s)$. Letting $t=s_{p}$ be the subterm of $s$ at that node, $s[n]=(t[m]+1) \bmod 2$.

We are now ready to establish a powerful theorem, which holds for all groupoid terms regardless of any conditions on the order or number of appearances of variables.

Theorem 3.4. Let $s$ and $t$ be any groupoid terms. Suppose the variable $x$ has an occurrence in $s$ where the path to that occurrence is $p$, and that $x$ has an occurrence in $t$ where the path to that occurrence is $q$. Then if $q$ is a proper initial substring of $p$, the terms $s$ and $t$ can be separated.

Proof. Let $s, t, x, p$ and $q$ be as above. By hypotheses, $p=q w$ for a nonempty string $w$. We let $\star$ be $\|1, q, 0\|+\|1, w, 1\|^{\prime}$. 
First consider the value of $t[0]$ for this $\star$. Since $\|1, w, 1\|^{\prime}$ does not have an assignment to $z[0],\|1, w, 1\|^{\prime}$ makes $t[0]=0$, and we can ignore it. As for $\|1, q, 0\|$, Lemma 3.2 gives $t[0]=t_{q}[1]=x[1]$. This implies that $\star$ sets $t[0]=x[1]$.

Now consider the value of $s[0]$ for the above $\star$. As in our calculation for $t[0]$, we have $s[0]=s_{q}[1]$. But now $s_{q}$ is a nontrivial subterm of $s$, so we compute $s_{q}[1]$. The operation $\|1, q, 0\|$ has no effect on $s_{q}[1]$, so we ignore it and just consider the effect of $\|1, w, 1\|^{\prime}$. It gives $s_{q}[1]=s_{q w}[1]+1$, by Lemma 3.3. Putting these together, we have $s[0]=s_{q}[1]=s_{q w}[1]+1=$ $s_{p}[1]+1=x[1]+1$. This shows that $s$ and $t$ always have different values in a finite groupoid, since it is always true that $s[0] \neq t[0]$.

Theorem 3.5. For all $k \geq 3$ there is a $k$-antiassociative finite groupoid.

Proof. It is enough to produce a finite groupoid that separates any two distinct $k$-ary ordered terms $s$ and $t$. Given any two distinct terms $s$ and $t$ with $k \geq 3$, we let $x_{m}$ be the leftmost variable on which $s$ and $t$ do not agree, in the sense that $\operatorname{path}_{s}\left(x_{i}\right)=\operatorname{path}_{t}\left(x_{i}\right)$ for all $i<m$, and $\operatorname{path}_{s}\left(x_{m}\right) \neq \operatorname{path}_{t}\left(x_{m}\right)$.

We claim that for any two such distinct $k$-ary terms $s$ and $t$, one of $\operatorname{path}_{s}\left(x_{m}\right)$ or $\operatorname{path}_{t}\left(x_{m}\right)$ is a proper initial substring of the other. The proof is by induction on $j$, where $j$ is the minimum of the lengths of $\operatorname{path}_{s}\left(x_{m}\right)$ and $\operatorname{path}_{t}\left(x_{m}\right)$. If $j=0$, then either $s=x_{m}$ or $t=x_{m}$. Without loss of generality, assume $s=x_{m}$. Then $\operatorname{path}_{s}\left(x_{m}\right)=\Lambda$. If $\operatorname{path}_{t}\left(x_{m}\right)$ is also $\Lambda$, we have $s=x_{m}=t$, a contradiction. So $\operatorname{path}_{t}\left(x_{m}\right) \neq \Lambda$, and $\operatorname{path}_{t}\left(x_{m}\right)$ has $\operatorname{path}_{s}\left(x_{m}\right)$ as a proper initial substring. This establishes the basis case.

For the induction step, suppose that the claim is true for $j-1$, and that we want to prove it for $j$. We have that $s=s_{l} \star s_{r}$, and $t=t_{l} \star t_{r}$. We have two cases, depending on where $x_{m}$ occurs.

If $x_{m}$ occurs in $s_{r}$, then $x_{m}$ also occurs in $t_{r}$ since $s_{l}=t_{l}$ because $s$ and $t$ agree for all $i<m$. But then $x_{m}$ is the leftmost variable on which $s_{r}$ and $t_{r}$ disagree, so one of $\operatorname{path}_{s_{r}}\left(x_{m}\right)$ and $\operatorname{path}_{t_{r}}\left(x_{m}\right)$ is a proper initial substring of the other, by the statement for $j-1$. Since $\operatorname{path}_{s}\left(x_{m}\right)$ and $\operatorname{path}_{t}\left(x_{m}\right)$ are obtained from these paths by adding $r$ to the start, one of them is also a proper initial substring of the other.

So suppose $x_{m}$ occurs in $s_{l}$. As in the previous paragraph, if $x_{m}$ occurred in $t_{r}$, we would get that $x_{m}$ occurred in $s_{r}$. Thus $x_{m}$ occurs in $t_{l}$. Then $x_{m}$ is the leftmost variable on which $s_{l}$ and $t_{l}$ disagree, and one of $\operatorname{path}_{s_{l}}\left(x_{m}\right)$ and path $_{t_{l}}\left(x_{m}\right)$ is a proper initial substring of the other. Adding $l$ to the start of these paths gives $\operatorname{path}_{s}\left(x_{m}\right)$ and $\operatorname{path}_{t}\left(x_{m}\right)$, so one of them is a proper initial substring of the other. This proves the claim. 
Now let distinct $k$-ary $s$ and $t$ with $k \geq 3$ be given. The claim gives us that one of $\operatorname{path}_{t}\left(x_{m}\right)$ and $\operatorname{path}_{s}\left(x_{m}\right)$ is a proper initial substring of the other. We apply Theorem 3.4, and obtain a finite groupoid that separates $s$ and $t$.

\section{Separating arbitrary groupoid terms}

We can generalize the questions of the previous section, by relaxing the condition that each variable appears once in every term in order of their indices.

As before, we can reduce everything to the problem of finding finite algebras that separate pairs of terms. (Theorem 4.3 uses free algebras to give us a condition for when infinite algebras exist that separate a pair of terms.)

If we try to separate the two groupoid terms $s(x, y)=x \star y$ and $t(x, y)=$ $y \star x$, we rapidly run into trouble. When $x=y$, both terms reduce to $x \star x$, so it is impossible to separate them in any groupoid. This trick of identifying variables can be applied whenever $s$ and $t$ have the same shape, which we can define rigorously as follows. Let $\chi$ be a distinguished variable symbol, that we agree to use nowhere else. Then we simply define the shape of a term $s\left(x_{1}, x_{2}, \ldots x_{k}\right)$ to be the term $s(\chi, \chi, \ldots \chi)$.

As an aside, note that we can easily make the term functions $x \star y$ and $y \star x$ not equal whenever $x \neq y$, for instance by letting $\star$ be - over $Z_{3}$. This prompts the following question, which we will not deal with further in this paper.

Question 4.1. Suppose that $s$ and $t$ are two terms of the same shape, and let $x_{1}, x_{2}, \ldots x_{k}$ be all the variables appearing in either of them. Given a set $S$ of equalities between variables in $\left\{x_{1}, x_{2}, \ldots x_{k}\right\}$, there is a function $\phi$ from $\{1,2, \ldots k\}$ into $\{1,2, \ldots k\}$ such that for each $i, \phi(i)$ is the least number such that the equality $x_{i}=x_{\phi(i)}$ can be deduced from equalities in $S$. Also let $s^{\prime}$ be the term that results when for all $i, x_{i}$ is replaced by $x_{\phi(i)}$ throughout $s$, and let $t^{\prime}$ be defined similarly.

Call a set of equalities $S$ between variables in $\left\{x_{1}, x_{2}, \ldots x_{k}\right\}$ identifying iff the terms $s^{\prime}$ and $t^{\prime}$ are the same. When is it possible to have a finite algebra where the term functions $s$ and $t$ are not equal whenever the values of their variables do not satisfy any identifying sets of equations?

From now on, we will focus on separating two groupoid terms of different shapes. Since we are now dealing with arbitrary terms, variables may occur more than once in a given term. For clarity, we will usually use primes to distinguish occurrences of a variable from the variable itself, so that $x^{\prime}$ might 
denote some particular occurrence of $x$. We will say that terms $s$ and $t$ are finitely separated whenever they are separated in some finite groupoid.

Observe that any groupoid term $s$ has a natural order to the occurrences of its variables, the order produced by an inorder transversal of the leaves of its full binary tree $T(s)$. We will always write terms by listing occurences of variables in this natural order. In this case, we call $x_{1}^{\prime}$ the leftmost variable occurrence in $s\left(x_{1}, \ldots\right)$. Each variable occurrence in $s$ corresponds to a leaf in $T(s)$, so occurrences of a given variable may be distinguished by their paths in $T(s)$. The leftmost variable occurrence in $s$ is then the only one with a path in $\{l\}^{*}$.

By the depth of an occurrence of a variable in the term $s$, we mean its height in $T(s)$. We will denote the depth in $s$ of the variable occurrence $x^{\prime}$ by $d_{s}\left(x^{\prime}\right)$. Note that this is the same as the length of the string $\operatorname{path}_{s}\left(x^{\prime}\right)$.

A naive intuition would be that terms $s$ and $t$ could not be separated when there were a number of variables occurring in one term and not the other. It is certainly true that having more variables of this sort gives more possibilities to assign values to them that would force $s$ and $t$ to be equal. For example, let $s$ be $(x \star y) \star z$, and let $t$ be $(x \star x) \star(x \star x)$. Letting $x$ have any fixed value, we assign $y:=x$ and $z:=x \star x$. Substituting these values in $s$, it becomes $(x \star x) \star(x \star x)$, which is $t$. So $s$ and $t$ can not be separated in any groupoid.

However, there are terms with only a single variable in common that can still be separated in a finite groupoid. For example, let $s$ be $x \star p$ and let $t$ be $(x \star y) \star q$, where $p$ and $q$ can be arbitrary terms on any variables. For the leftmost occurrences of $x$, we have $\operatorname{path}_{s}(x)=l$ and $\operatorname{path}_{t}(x)=l l$. So Theorem 3.4 gives a finite groupoid that separates $s$ and $t$.

To continue our investigation, we need the following extension of Theorem 3.4, which requires further definitions to state. If $s$ and $t$ are groupoid terms and $y$ and $z$ are variables, we say that $y$ occurs above $z$ if there are occurrences $y^{\prime}$ of $y$ and $z^{\prime}$ of $z$ so that either $\operatorname{path}_{s}\left(y^{\prime}\right)$ is a initial substring of $\operatorname{path}_{t}\left(z^{\prime}\right)$ or $\operatorname{path}_{t}\left(y^{\prime}\right)$ is a initial substring of $\operatorname{path}_{s}\left(z^{\prime}\right)$. In this situation, we also say that the occurrence $y^{\prime}$ is above the occurrence $z^{\prime}$. Similarly, $y$ occurs strictly above $z$ if there are occurrences $y^{\prime}$ of $y$ and $z^{\prime}$ of $z$ so that either $\operatorname{path}_{s}\left(y^{\prime}\right)$ is a proper initial substring of $\operatorname{path}_{t}\left(z^{\prime}\right)$ or $\operatorname{path}_{t}\left(y^{\prime}\right)$ is a proper initial substring of $\operatorname{path}_{s}\left(z^{\prime}\right)$.

We say that terms $s$ and $t$ have a cycle if there is a sequence of variables $y_{0}, y_{1}, \ldots y_{m-1}$ where $y_{0}$ occurs above $y_{1}, y_{1}$ occurs above $y_{2}$, and so on, ending with $y_{m-1}$ occurring above $y_{0}$, where at least one of these occurrences is strictly above the other. The hypothesis of Theorem 3.4 is that a single variable $x$ occurs above itself, so that $s$ and $t$ have a cycle of length 1 , where the sequence $y_{0}, y_{1}, \ldots y_{m-1}$ is just $x$. Our next theorem extends this result 
to cycles of arbitrary length.

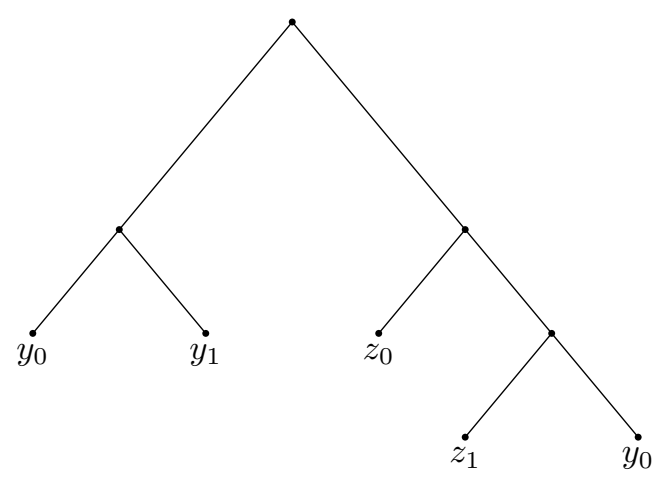

$s=\left(y_{0} \star y_{1}\right) \star\left(z_{0} \star\left(z_{1} \star y_{0}\right)\right)$

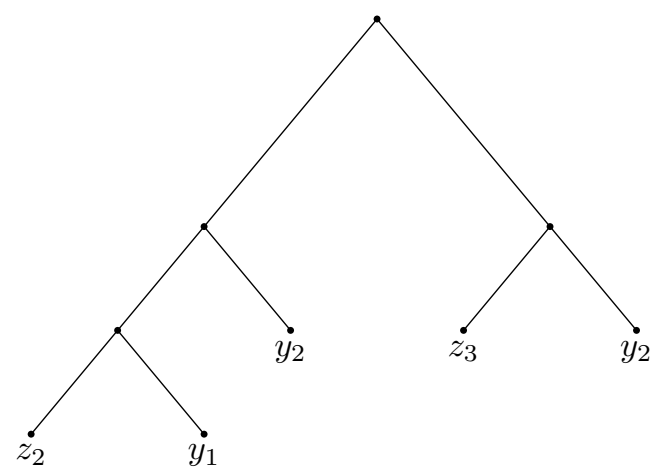

$t=\left(\left(z_{2} \star y_{1}\right) \star y_{2}\right) \star\left(z_{3} \star y_{2}\right)$

Figure 2: Two terms with a cycle

This proof will be easier to follow if we have an example for reference. It may be useful to refer back to this example while reading the proof, as some of the notation it uses is defined in the proof. Figure 2 shows a cycle $y_{0} y_{1} y_{2}$ of length 3 , where $s=\left(y_{0} \star y_{1}\right) \star\left(z_{0} \star\left(z_{1} \star y_{0}\right)\right)$ and $\left.t=\left(\left(z_{2} \star y_{1}\right) \star y_{2}\right) \star\left(z_{3} \star y_{2}\right)\right)$. Matching the notation of the coming theorem, we use superscripts of $u$ and $d$ (for "up" and "down") to label the distinct occurrences of variables in the cycle, as shown in Table1,

\begin{tabular}{c|c|c|l|l|l} 
index & occurrence & term & path & $p_{i}$ & $q_{i}$ \\
\hline 0 & $y_{0}^{u}$ & $s$ & $l l$ & $l l$ & \\
& $y_{1}^{d}$ & $t$ & $l l r$ & & $r$ \\
\hline 1 & $y_{1}^{u}$ & $s$ & $l r$ & $l r$ & \\
& $y_{2}^{d}$ & $t$ & $l r$ & & $\Lambda$ \\
\hline 2 & $y_{2}^{u}$ & $t$ & $r r$ & $r r$ & \\
& $y_{0}^{d}$ & $s$ & $r r r$ & & $r$
\end{tabular}

Table 1: Occurrences in a cycle

In the cycle, $y_{0}$ is strictly above $y_{1}$, since the occurrence $y_{0}^{u}$ in $s$ has path $l l$, which is an initial substring of $l l r$, the path in $t$ of the occurrence $y_{1}^{d}$. And $y_{1}$ is above (but not strictly above) $y_{2}$, since the occurrence $y_{1}^{u}$ in $s$ has path $l r$, which is a (non-proper) initial substring of $l r$, the path in $t$ of the occurrence $y_{2}^{d}$. Finally, $y_{2}$ is strictly above $y_{0}$, since the occurrence $y_{2}^{u}$ in $t$ has path $r r$, which is an initial substring of $r r r$, the path in $s$ of the occurrence $y_{0}^{d}$. 
The theorem also defines relations $\sim$ and $\approx$ on the index set, which is $I=\{0,1,2\}$ in our example. We have $1 \sim 2$, since $y_{1}^{u}$ is not strictly above $y_{2}^{d}$. The relation $\approx$ is the equivalence relation generated by $\sim$, so its classes are $\{0\}$ and $\{1,2\}$. The function $f$ that takes each $i \in I$ to the least element in its $\approx$ class has $f(0)=0$ and $f(1)=f(2)=1$. Finally, the operation $\star^{\prime}$ is $\|3, l l, 0\|+\|4, l r, 1\|+\|4, r r, 2\|+\|4, r, 3\|^{\prime}+\|3, r, 4\|$. The reader can verify that this operation makes $s[0]+s[1]+s[2]=y_{0}[3]+y_{1}[4]+\left(z_{1} \star^{\prime} y_{0}\right)[4]=$ $y_{0}[3]+y_{1}[4]+y_{0}[3]=y_{1}[4]$, where the last step follows since we are adding values modulo 2. Similarly, $t[0]+t[1]+t[2]=\left(z_{2} \star^{\prime} y_{1}\right)[3]+y_{2}[4]+y_{2}[4]=$ $y_{1}[4]+1+y_{2}[4]+y_{2}[4]=y_{1}[4]+1$, which always has a different value.

Theorem 4.2. Let $s$ and $t$ be groupoid terms which have a cycle. Then $s$ and $t$ are separated in a finite groupoid.

Proof. Let $s$ and $t$ be terms with a cycle as above. So we have a sequence of variables $y_{0}, y_{1}, \ldots y_{m-1}$ where $y_{0}$ occurs above $y_{1}, y_{1}$ occurs above $y_{2}$, and so on, ending with $y_{m-1}$ occurring above $y_{0}$. We may assume that this cycle has minimal length $k$ for all cycles of $s$ and $t$, and that $k \geq 2$ since cycles of length 1 are covered by Theorem 3.4. This implies that all of the variables $y_{i}$ are distinct. We also adopt the convention that our subscripts are calculated modulo $k$, so that $y_{k}$ is the same as $y_{0}$.

Each of the $y_{i}$ has two occurrences in the cycle. For each $i$, let $y_{i}^{u}$ be the occurrence of $y_{i}$ that is above an occurrence of $y_{i+1}$, and let $y_{i}^{d}$ be the occurrence of $y_{i}$ that is below an occurrence of $y_{i-1}$. A given occurrence $y^{\prime}$ of a variable may be either in the term $s$ or in the term $t$.

We denote whichever of $s$ and $t$ an occurrence $y^{\prime}$ is in by $\operatorname{term}\left(y^{\prime}\right)$. We will then write $\operatorname{path}\left(y^{\prime}\right)$ to denote the path of $y^{\prime}$ in $\operatorname{term}\left(y^{\prime}\right)$. Note that term $\left(y_{i}^{u}\right) \neq$ $\operatorname{term}\left(y_{i+1}^{d}\right)$ for all $i, \operatorname{since} \operatorname{path}\left(y_{i}^{u}\right)$ is an initial substring of $\operatorname{path}\left(y_{i+1}^{d}\right)$ and $y_{i} \neq y_{i+1}$.

We will denote $\operatorname{path}\left(y_{i}^{u}\right)$ by $p_{i}$. And $\operatorname{since} \operatorname{path}\left(y_{i+1}^{d}\right)$ has $p_{i}$ as an initial substring, we will write it as the concatenation $p_{i} q_{i}$, where $q_{i}$ is possibly $\Lambda$.

We claim that none of the $p_{i}$ is an inital substring of any of the others. For suppose $i \neq j$ and $p_{i}$ is an initial substring of $p_{j}$. Since $y_{j}^{u}$ corresponds to a leaf of $T\left(\operatorname{term}\left(y_{j}^{u}\right)\right)$, we must have $\operatorname{term}\left(y_{i}^{u}\right) \neq \operatorname{term}\left(y_{j}^{u}\right)$. Now consider $y_{j+1}^{d}$. We have that $\operatorname{term}\left(y_{j+1}^{d}\right) \neq \operatorname{term}\left(y_{j}^{u}\right)$, so $\operatorname{term}\left(y_{j+1}^{d}\right)=\operatorname{term}\left(y_{i}^{u}\right)$. We also have that $p_{i}$ is an initial substring of $p_{j}$, which is an initial substring of $p_{j} q_{j}=\operatorname{path}\left(y_{j+1}^{d}\right)$. In $T\left(\operatorname{term}\left(y_{i}^{u}\right)\right)$, this would place the leaf corresponding to the occurrence $y_{j+1}^{d}$ below the leaf corresponding to $y_{i}^{u}$. The only way this could happen is if $y_{i}^{u}=y_{j+1}^{d}$. So $i=j+1$, and $y_{i}^{u}=y_{i}^{d}$. But then $\operatorname{term}\left(y_{i-1}^{u}\right) \neq$ $\operatorname{term}\left(y_{i}^{d}\right)=\operatorname{term}\left(y_{i}^{u}\right) \neq \operatorname{term}\left(y_{i+1}^{d}\right)$, so $y_{i-1}^{u}$ and $y_{i+1}^{d}$ are occurrences in the same term. Now path $\left(y_{i-1}^{u}\right)$ in an initial substring of $\operatorname{path}\left(y_{i}^{d}\right)=\operatorname{path}\left(y_{i}^{u}\right)$, which is an initial substring of $\operatorname{path}\left(y_{i+1}^{d}\right)$, implying that both $\operatorname{path}\left(y_{i-1}^{u}\right)$ and 
$\operatorname{path}\left(y_{i+1}^{d}\right.$ label the same leaf of the tree they are in. So $i-1=i+1=j$, and our cycle consists of just $y_{i}$ and $y_{j}$, with $y_{i}^{u}=y_{i}^{d}$ and $y_{j}^{u}=y_{j}^{d}$. This is a contradiction, since at least one variable occurrence in a cycle must be strictly above the next occurrence. The claimi s established.

Without loss of generality, assume that the occurrence $y_{0}^{u}$ is strictly above $y_{1}^{d}$, so $\operatorname{path}\left(y_{1}^{d}\right)$ is $p_{0} q_{0}$ where $q_{0} \neq \Lambda$. Let $I=\{0,1,2, \ldots k-1\}$ be our set of indices for the $y_{i}$, and let $N$ be $\left\{i \in I: q_{i} \neq \Lambda\right\}$ So $0 \in N$.

Define the relation $\sim$ on $I$ by $i \sim j$ iff $j=(i+1) \bmod k$ and $q_{i}=\Lambda$, and let $\approx$ be the equivalence relation generated by $\sim$. Intuitively, the classes of $\approx$ are runs of consecutive indices, with each class ending at an element of $N$.

Finally, define $f: I \rightarrow I$ by letting $f(i)$ the least element of the $\approx$ equivalence class of $i$. This gives us that $f(i)=f(i+1)$ when $q_{i}=\Lambda$. (We usually have $f(i) \neq f(i+1)$ when $q_{i} \neq \Lambda$. The one exception is when only one of the $q_{j}$ is not $\Lambda$, so $i$ and $i+1$ are related by $\approx$ the long way around the cycle.)

Now we define the groupoid operation $\star$ to be the sum $\left[\left\|k+f(0), p_{0}, 0\right\|+\right.$ $\left.\left\|k+f(1), p_{1}, 1\right\|+\cdots\left\|k+f(k-1), p_{k-1}, k-1\right\|\right]+\sum_{i \in N}\left\|k+f(i+1), q_{i}, k+f(i)\right\|$ The groupoid operation $\star^{\prime}$ will be $\star+\left\|k+f(1), q_{0}, k+f(0)\right\|^{\prime}-\left\|k+f(1), q_{0}, k+f(0)\right\|$, a slight variation of $\star$ where the operation $\left\|k+f(1), q_{0}, k+f(0)\right\|$ is replaced with the tweaked operation $\left\|k+f(1), q_{0}, k+f(0)\right\|^{\prime}$, while all of the other operations remain unchanged.

We will show that in the groupoid with operation $\star$, that the sum modulo 2 of $s[0]+s[1]+\ldots s[k-1]$ will always equal the sum modulo 2 of $t[0]+$ $t[1]+\ldots t[k-1]$. Then we will confirm that in the groupoid with operation $\star^{\prime}$, the two corresponding sums of components will differ. This difference will be caused by the tweaked operation $\left\|k+f(1), q_{0}, k+f(0)\right\|^{\prime}$, which will only produce an effect in the final output in $\operatorname{term}\left(y_{1}^{d}\right)$, the term where the occurrence $y_{1}^{d}$ lies. For the moment, we will be working with the operation $\star$.

First, we establish that for any $y_{i}$, the value of the $i$-th component of $\operatorname{term}\left(y_{i}^{u}\right)$ will be $y_{i}[k+f(i)]$. Without loss of generality, let term $\left(y_{i}^{u}\right)$ be $s$. The only summand of $\star$ that assigns a value to $s[i]$ is $\left\|k+f(i), p_{i}, i\right\|$, so $s[i]$ will have the value it assigns. We apply Lemma 3.2, and get that $s[i]$ is equal to $r[k+f(i)]$, where $r$ is the subterm of $s$ with path $p_{i}$. In this case, $r=y_{i}$, so $s[i]=r[k+f(i)]=y_{i}[k+f(i)]$, as desired.

Given any $i$, we let $j=i+1 \bmod k$. We now show that for any $y_{j}$, the value of the $i$-th component of $\operatorname{term}\left(y_{j}^{d}\right)$ is also $y_{j}[k+f(j)]$. Without loss of generality, let term $\left(y_{j}^{d}\right)$ be $t$. As before, $t[i]$ will be equal to $r[k+f(i)]$, where $r$ is the subterm of $t$ with path $p_{i}$. We now have two cases. If $i \notin N$, then $q_{i}=\Lambda$ and $\operatorname{path}\left(y_{j}^{d}\right)=p_{i}$, making $r=y_{j}$ and $t[i]=y_{j}[k+f(i)]=y_{j}[k+f(j)]$ since $i \sim j$. So assume $i \in N$. Then $r$ is a nontrivial subterm of $t$, where 
$\operatorname{path}_{r}\left(y_{j}^{d}\right)$ is $q_{i}$. The only term in $\star$ that assigns a value to $r[k+f(i)]$ is $\left\|k+f(i+1), q_{i}, k+f(i)\right\|$, so $r[k+f(i)]$ is $y_{j}[k+f(i+1)]=y_{j}[k+f(j)]$, as desired.

For each $i$, we do not know which of $s$ and $t$ the occurrences $y_{i}^{u}$ and $y_{i+1}^{d}$ are in. This turns out not to be an obstacle, since we do know that $y_{i}^{u}$ and $y_{i+1}^{d}$ occur in different terms. Working modulo 2, we have that $s[0]+$ $s[1]+\cdots s[k-1]+t[0]+t[1]+\cdots t[k-1]=\left[y_{0}[k+f(0)]+y_{1}[k+f(1)]+\right.$ $\left.\cdots y_{k-1}[k+f(k-1)]\right]+\left[y_{1}[k+f(1)]+y_{2}[k+f(2)]+\cdots y_{k}[k+f(k)]\right]$, where the second group on the right hand side comes from the $y_{j}^{d}$. But the latter expression is equal to $2\left[y_{0}[k+f(0)]+\cdots y_{k-1}[k+f(k-1)]\right]=0$ modulo 2 . Since $s[0]+\cdots s[k-1]$ and $t[0]+\cdots t[k-1]$ sum to 0 , they have the same parity.

Now we turn to the groupoid with operation $\star^{\prime}$, and consider the effect of the tweaked operation $\left\|k+f(1), q_{0}, k+f(0)\right\|^{\prime}$. The reader may verify that everything works as before, except in the calculation of the 0 -th component of $\operatorname{term}\left(y_{1}^{d}\right)$. As before, we may assume that $s$ is term $\left(y_{1}^{d}\right)$. We then get $s[0]=r[k+f(0)]$, where $r[k+f(0)]$ is found using $\left\|k+f(1), q_{0}, k+f(0)\right\|^{\prime}$. This makes $r[k]=y_{1}[k+f(1)]+1 \bmod 2$, giving $s[0]=y_{1}[k+f(1)]+1$ $\bmod 2$. This in turn changes the parity of $s[0]+s[1]+\cdots s[k-1]$ in whichever term we are calling $s$, as desired.

As in Theorem 3.4, this yields a finite groupoid that separates $s$ and $t$.

We would like to have a nice characterization of which pairs of groupoid terms can be separated in a finite groupoid. So we will also investigate when it is impossible to separate a pair of terms in any groupoid.

We need a bit of preliminary material on free algebras. A more detailed exposition may be found in [3]. We use $\mathbf{G}$ for the class of all groupoids, and let $F_{\mathbf{G}}\left(y_{0}, y_{1}, \ldots y_{n-1}\right)$ denote the free groupoid with generators $y_{0}, y_{1}, \ldots y_{n-1}$. The key feature of $F_{\mathbf{G}}\left(y_{0}, y_{1}, \ldots y_{n-1}\right)$ is that it has the Universal Mapping Property for the class of groupoids. If $G$ is any groupoid with elements $g_{0}, g_{1}, \ldots g_{n-1}$, then there is a unique homomorphism $\phi$ from $F_{\mathbf{G}}\left(y_{0}, y_{1}, \ldots y_{n-1}\right)$ into $G$ where $\phi\left(y_{i}\right)=g_{i}$ for all $i$.

Theorem 4.3. Let $s$ and $t$ be groupoid terms, each on a set of variables that is a subset of $\left\{y_{0}, y_{1}, \ldots y_{n-1}\right\}$. Then the following are equivalent.

1. $s$ and $t$ are separated in some groupoid.

2. $s$ and $t$ are separated in $F_{\mathbf{G}}\left(y_{0}, y_{1}, \ldots y_{n-1}\right)$.

3. $s$ and $t$ are separated in $F_{\mathrm{G}}(x)$, the free groupoid on one variable. 
Proof. Let $s$ and $t$ be groupoid terms with all their variables in $\left\{y_{0}, y_{1}, \ldots y_{n-1}\right\}$. It is clear that (2) implies (1). To see that (3) implies (2), suppose that (2) fails. Then there are terms $h_{0}, h_{1}, \ldots h_{n-1}$ in $F_{\mathbf{G}}\left(y_{0}, y_{1}, \ldots y_{n-1}\right)$ with $s\left(h_{0}, h_{1}, \ldots h_{n-1}\right)=t\left(h_{0}, h_{1}, \ldots h_{n-1}\right)$. The $h_{i}$ are all generated from $\left\{y_{0}, \ldots y_{n-1}\right\}$ by repeatedly using the groupoid operation. Now consider the homomorphism $\phi$ from $F_{\mathbf{G}}\left(y_{0}, y_{1}, \ldots y_{n-1}\right)$ into $F_{\mathbf{G}}(x)$ that takes all of the $y_{i}$ to $x$. Denoting the image of each $h_{i}$ by $h_{i}^{\prime}$, we have that $s\left(h_{0}^{\prime}, h_{1}^{\prime}, \ldots h_{n-1}^{\prime}\right)=$ $t\left(h_{0}^{\prime}, h_{1}^{\prime}, \ldots h_{n-1}^{\prime}\right)$ in $F_{\mathbf{G}}(x)$, so $(3)$ fails.

To see (1) implies (3), assume that (3) fails. So we have $f_{0}, f_{1}, \ldots f_{n-1} \in$ $F_{\mathbf{G}}(x)$ with $s\left(f_{0}, f_{1}, \ldots f_{n-1}\right)=t\left(f_{0}, f_{1}, \ldots f_{n-1}\right)$. Letting $G$ be any groupoid, we pick any $c \in G$, and consider the homomorphism $\phi$ from $F_{\mathbf{G}}(x)$ to $G$ that takes $x$ to $c$. Letting the image of each $f_{i}$ be $f_{i}^{\prime}$, we have that $s\left(f_{0}^{\prime}, f_{1}^{\prime}, \ldots f_{n-1}^{\prime}\right)=t\left(f_{0}^{\prime}, f_{1}^{\prime}, \ldots f_{n-1}^{\prime}\right)$ in $G$, so (1) fails.

The free groupoid $F_{\mathbf{G}}(x)$ is easy to work with, since all of its elements may be viewed as groupoid terms in the single variable $x$. Terms $s$ and $t$ are separated in $F_{\mathbf{G}}(x)$ iff there are no terms $f_{0}(x), f_{1}(x), \ldots f_{n-1}(x) \in F_{\mathbf{G}}(x)$ that can be substituted for the variables of $s$ and $t$ to yield $s\left(f_{0}(x), f_{1}(x), \ldots f_{n-1}(x)\right)=$ $t\left(f_{0}(x), f_{1}(x), \ldots f_{n-1}(x)\right)$.

This relates to the notion of unification of terms, which has been extensively studied in computer science. The introduction of the topic was by Herbrand, in [5]. Modern work was pioneered by Robinson, in 9]. Good survey articles are by Knight (in [7]) and Jouannaud and Kirchner (in [6]). Consider two terms $s\left(x_{0}, \ldots x_{m-1}\right)$ and $t\left(y_{0}, \ldots y_{n-1}\right)$. The terms are unifiable if there are terms $r_{0}, \ldots r_{m-1}$ and $u_{0}, \ldots u_{n-1}$ so that substituting the $r_{i}$ for the $x_{i}$ in $s$ and the $u_{j}$ for the $y_{j}$ in $t$ makes the two resulting terms identical, and the corresponding substitution is a unification. In other words, the terms $s$ and $t$ can be unified iff they can not be separated in a free algebra. In view of the previous theorem, two terms can not be unified iff there is a groupoid where they are separated.

Algorithms to see whether or not two terms $s$ and $t$ can be unified are discussed in detail in [7] and [6]. An inefficient but effective method for groupoid terms is to use the following rules for generating sets of statements, starting with the statement $s=t$. In each rule, a,b,c and d are terms, while $\mathrm{x}$ and $\mathrm{y}$ are variables.

1. (Decompose) From $a \star b=c \star d$ deduce $a=c$ and $b=d$.

2. (Coalesce) If we have $x=y$, deduce the results of replacing every $x$ in our set of statements with a $y$.

3. (Check) From $x=a$, deduce False if $x$ occurs in the term $a$. 
4. (Eliminate) From $x=a$, deduce the results of replacing every $x$ in our set of statements with the term $a$, provided $x$ does not occur in $a$.

One may simply apply all the rules repeatedly, until no more statements are deduced. If False is ever deduced, the original terms $s$ and $t$ can not be unified. Otherwise, a unifying set of substitutions will be deduced. In practice, one may be more targeted in applying the rules and reach False or unifying substitutions more rapidly.

For example, consider $s=(x \star y) \star(z \star y)$ and $t=z \star((x \star y) \star(x \star x))$. We will use the algorithm to see if they can be unified. We start with $s=t$. Using Decompose, we obtain $z=x \star y$ and $z \star y=(x \star y) \star(x \star x)$. Applying Decompose again to the last statement, we get $z=x \star y$ (a duplicate) and $y=x \star x$. Applying Eliminate using $y=x \star x$ to $z=x \star y$, we get $z=x \star(x \star x)$. We have found a set of unifying substitutions. Letting $y=x \star x$ and $z=x \star(x \star x)$ in $s$ and $t$, both become $(x \star(x \star x)) \star((x \star(x \star x)) \star(x \star x))$.

Here is an example with a cycle. In view of Theorem 4.2 , it will be no surprise that this is an obstacle to unification. Let $s=(x \star y) \star(z \star w)$ and let $t=((w \star u) \star x) \star((y \star v) \star z)$. Repeatedly applying Decompose, we get $x=w \star u, y=x, z=y \star v$ and $w=z$. Applying Coalesce, we get $x=z \star u$ and $z=x \star v$. Applying Eliminate gives $x=(x \star v) \star u$, and applying Check gives False. Our applications of Coalesce and Eliminate acted to reduce the length of the original cycle. Using the notation of Theorem 4.2, this cycle had $y_{0}^{u}$ the $x$ in $s, y_{0}^{d}$ the $w$ in $t, y_{1}^{u}$ the $w$ in $s, y_{1}^{d}$ the $z$ in $t, y_{2}^{u}$ the $z$ in $s$, $y_{2}^{d}$ the $y$ in $t, y_{3}^{u}$ the $y$ in $s$, and $y_{3}^{d}$ the $x$ in $t$.

There are pairs of terms without a cycle which still can not be unified. For example, let $s=(x \star y) \star(z \star y)$ and let $t=z \star((y \star y) \star(x \star x))$. Working left to right, we see that $x$ and $y$ occur below $z, y$ occurs below $z$ and $x$ occurs below $y$. This is consistent with the ordering $x<y<z$. Since there is a consistent ordering of the variables like this, there are no cycles. However, $s$ and $t$ can not be unified. Applying Decompose repeatedly gives $z=x \star y$, $z=y \star y$ and $y=x \star x$. Then applying Eliminate to the first two gives $x \star y=y \star y$, after which Decompose gives $x=y$. Finally, Eliminate gives $x=x \star x$, and Check gives False.

Although Theorem 4.2 does not apply to this last example, we had no problem separating the terms using a similar construction. Letting $\star^{\prime}=$ $\|3, l, 0\|+\|3, r l, 1\|+\|4, r r, 2\|+\|4, l, 3\|+\|4, l, 4\|^{\prime}$, we calculate $s[0]+s[1]+$ $s[2]=(x \star y)[3]+z[3]+y[4]=x[4]+z[3]+y[4]$, while $t[0]+t[1]+t[2]=$ $z[3]+(y \star y)[3]+(x \star x)[4]=z[3]+y[4]+x[4]+1$, which has the opposite parity.

Based on many examples similar to the above, we make the following conjecture. 
Conjecture 4.4. Whenever two groupoid terms can be separated in an infinite groupoid, they can also be separated in a finite groupoid.

\section{References}

[1] Milton Braitt and Donald Silberger, Subassociative groupoids, Quasigroups and Related Systems 14 (2006), 11 - 26.

[2] Milton Braitt, David Hobby and Donald Silberger, Completely dissociative groupoids, Mathematica Bohemica, 137 (2012), No. 1, pp. 79-97.

[3] Stanley N. Burris and H. P. Sankappanavar, "A Course in Universal Algebra". Springer-Verlag, 1981. ISBN 3-540-90578-2. (Also freely available online at http://www.thoralf.uwaterloo.ca/htdocs/ualg.html)

[4] Chang and Kiesler, "Model Theory". Elsevier Science Publishers V.P., 1992. ISBN 0444880542

[5] Herbrand, J., Recherches sur la théorie de la Démonstration, Travaux de la Société des Sciences et des Lettres de Vorsovie, Classe III 33(128), 1930.

[6] Jouannaud, J.P., Kirchner, C., Solving equations in abstract algebras: A rule-based survey of unification, in J.L. Lassez and G. Plotkin, eds, Computational Logic: Essays in Honor of A. Robinson, MIT Press, Cambridge, MA, 1991.

[7] Knight, K., Unification: A multidisciplinary survey, Association for Computing Machinery, Computing Surveys 21(1), 1989, 93-124.

[8] Donald Knuth. The art of computer programming vol 1. Fundamental Algorithms, Third Edition. Addison-Wesley, 1997. ISBN 0-201-89683-4.

[9] Robinson, J.A., A machine oriented logic based on the resolution principle, Journal of the ACM 12(1), 1965, 23-41.

[10] Stanley, Richard P., Enumerative combinatorics. Vol. 2, Cambridge Studies in Advanced Mathematics, 62 (1999), Cambridge University Press, ISBN 978-0-521-56069-6.

\section{Addresses}

${ }^{1}$ Milton Braitt. Departamento de Matemática, Universidade Federal de Santa Catarina, Cidade Universitária, Florianópolis, SC 88040-900, Brasil 
Email: MSBraitt@mtm.ufsc.br

${ }^{2}$ David Hobby and Donald Silberger. Department of Mathematics, State University of New York, New Paltz NY 12561 - U.S.A.

Emails: hobbyd@newpaltz.edu or silbergd@newpaltz.edu

2010 Mathematics Subject Classification: Primary: 20N02, 08A99 Secondary: 68Q99, 68T15

Keywords: groupoids, non-associative, unification 